Erschienen in: Gardt, Andreas/Mattheier, Klaus J./Reichmann, Oskar (Hrsg.): Sprachgeschichte des Neuhochdeutschen. Gegenstände, Methoden, Theorien. Tübingen: Niemeyer, 1995. S. 199-226.

(Reihe Germanistische Linguistik 154)

ULRIKE HASS-ZUMKEHR

\title{
Daniel Sanders und die Historiographie der Germanistik
}

1. Das Beispiel Daniel Sanders (1819-1897)

2. Probleme der Historiographie der Germanistik

3. Alternative Überlegungen

4. Literatur

Die zwei Gegenstände, die in der Formulierung meines Themas benannt werden, stehen in einem Verhältnis zueinander, das ich problematisieren will, weil es in exemplarischer Weise Mängel und Gefahren der bisherigen Fachgeschichtsschreibung erkennen läßt. Daniel Sanders kommt in den Darstellungen zur Geschichte der Anfänge der Germanistik im 19. Jahrhundert nämlich entweder gar nicht vor oder dient dort ausschließlich als Hintergrundfolie des Wirkens der Gründerväter Grimm.

Ich werde nachfolgend sein Wirken und seine Rolle in der Geschichte der Germanistik skizzieren und anschließend danach fragen, warum er und andere Sprachforscher in der bisherigen Historiographie weitgehend vernachlässigt worden sind. Zum Schluß stelle ich alternative Überlegungen zu den Prinzipien der Fachgeschichte, insbesondere zur Bestimmung ihres Gegenstands, vor.

\section{Das Beispiel Daniel Sanders (1819-1897)}

Wer Daniel Sanders kennt, kennt ihn als eine der „zwei Spinnen“, die über den „Wortgarten“ des Grimmschen Wörterbuchs „gekrochen" sind und dort ihr „Gift ausgelassen“ haben. Mit diesen Worten hatte Jacob Grimm in seiner Wörterbuchvorrede von 1854 Sanders und einen weiteren Kritiker, Christian Friedrich Ludwig Wurm, polemisch charakterisiert. Obwohl er keine Namen nannte, wußte jeder, wer gemeint war, so groß war das Aufsehen, das diese beiden mit ihren Kritiken in- und außerhalb der Fachwelt erregt hatten. War Sanders gestern deshalb ein schamloser Geselle, so gilt er heute als ein ,germanistischer Außenseiter“ (Kirkness 1991, 299). Er war der erste jüdische Sprachgermanist überhaupt ${ }^{1}$ und womöglich der einzige freimütig-unerschrockene Kritiker der hochberühmten und -gelehrten Brüder; der einzige, der den Absolutheitsanspruch der historischen Schule in bezug auf Erforschung und Darstellung der

I Siehe die kompendiöse Zusammenstellung von Katznelson $(1959,342)$. 
deutschen Sprache nicht akzeptieren wollte und hinsichtlich der Gegenwartssprache einen alternativen Ansatz entwickelte. Jedenfalls ist es ihm nicht gut bekommen, das Terrain der germanistischen Wissenschaft zu betreten. Nach herrschender Meinung hätte er Buchhändler, Verleger oder Journalist werden sollen, wie viele andere Vertreter jener bildungsbesessenen, akademisch qualifizierten, emanzipationshungrigen und mehr oder weniger assimilationsbereiten jüdischen Generation der „Enkel“ Moses Mendelssohns.

Die Rolle, die Sanders für die Geschichte der Germanistik und insbesondere für die deutsche Lexikographie spielen sollte, geht auf ein eigentlich ganz unspektakuläres Ereignis zurück. Der Schulleiter einer im mecklenburgischen Abseits gelegenen Bürgerschule, die von der jüdischen Gemeinde getragen und für Schüler aller Konfessionen offen war, hatte neben seiner Bildungsarbeit, deren Erfolge über die Landesgrenzen hinaus bekannt geworden waren, auch wissenschaftlich gearbeitet. Sehr viele Gymnasiallehrer taten dies ihrem Selbstverständnis gemäß und publizierten die Ergebnisse eigenständiger Forschung in Schulprogrammen und diversen Fach- und Bildungszeitschriften.

Sanders hatte zwar ein sehr breites, Naturwissenschaften und Sprachen umfassendes Studium absolviert, doch lag sein Interessenschwerpunkt trotz mathematischer Dissertation von Anfang an bei den Sprachen, und zwar zunächst bei den klassischen Sprachen Griechisch und Latein. Unter dem Einfluß des nachwehenden Philhellenismus sammelte und edierte er zunächst neugriechische „Volkspoesie“, später auch Volkslieder und Märchen in der Mundart seiner mecklenburg-strelitzischen Heimat. Die romantische Sicht der Mutterund Volkssprache erfaßte ihn genauso wie viele nichtjüdische Philologen seiner Generation. Daß die Muttersprache seines Großvaters mit ziemlicher Wahrscheinlichkeit ${ }^{2}$ noch Jiddisch war, spielte bei dem rasanten Bildungsfortschritt der deutschen Juden jener Zeit keine hemmende, eher eine anspornende Rolle. Sanders dachte, fühlte und sprach Deutsch so gut wie jeder andere Patriot, ob katholischer, evangelischer oder ,israelitischer Konfession“.

Oberlehrer Sanders verfolgte die Aktivitäten der germanistischen Philologie mit großem Interesse, sah er doch gewisse Parallelen zur neugriechischen Philologie, die sich ebenfalls in ein ideologisch bewußtes Verhältnis zu Freiheit und Einheit des eigenen Volks setzte. Besonders der Plan zu einem deutschen Nationalwörterbuch, für den seit 1837 die Brüder Grimm öffentlich warben, bewegte ihn sehr. Sanders folgte ihrem öffentlichen Aufruf und schickte 1844/ 45 Jacob Grimm, was er gesammelt hatte, jedoch ohne die erhoffte Resonanz zu finden (vgl. Kirkness 1980). Grimm maß dem Belegmaterial, das gegenwartssprachbezogen gewesen sein muß, keine besondere Bedeutung bei. Sanders erwartete die ersten Lieferungen des Deutschen Wörterbuchs mit großer Spannung. Das Ergebnis enttäuschte ihn in mehrfacher Hinsicht und reizte ihn

2 D. h. nach allgemeiner Kenntnis des Verhältnisses, das die deutschen Juden zur deutschen Sprache vor und während der Emanzipationsepoche entwickelten. 
zum Widerspruch, zum wissenschaftlichen Disput. Ganz im Rahmen seiner bisherigen Publikationsgewohnheiten legte Sanders 1852/53 in zwei Folgen eine umfangreiche und detaillierte Kritik vor: Das deutsche Wörterbuch von Jacob Grimm und Wilhelm Grimm kritisch beleuchtet. (Hamburg: Hoffmann und Campe 1851; 2. Heft 1853). Für Grimm und die gesamte historische Schule der germanistischen Philologie war diese „kecke That" ein Schock, der heute noch nachhallt. Mit öffentlicher Kritik hatten Jacob und Wilhelm Grimm, Heroen der Wissenschaft und der Nation, nicht gerechnet und sie ließen sich auch nicht auf den Disput ein, den Sanders sich vielleicht nach dem Vorbild jener um Wahrheit ringenden Geistesschlachten erträumte, wie sie die Aufklärung, etwa Lessing contra Goeze, hervorgebracht hatte.

Es war Zufall, daß gerade 1852, im Jahr dieser aufsehenerregenden Kritik an den germanistischen „Göttern“, Oberlehrer Sanders seinen bisherigen Hauptberuf aufgeben mußte. Der jüdischen Schule wurde ganz plötzlich ihre Prüfungsund damit Existenzberechtigung entzogen, als die christliche Schule jüdische Schüler - und in begrenzter Zahl auch jüdische Lehrer - aufzunehmen begann, ein Vorgang, der nicht untypisch für die deutsch-jüdische Schulgeschichte jener Jahrzehnte war. Und dennoch spielte ein persönliches Schicksalsmoment für Sanders hinein: Er war vier Jahre zuvor einer der Aktiven in der Bewegung für die Verfassungsreform und die Verbesserung der bürgerlichen Rechte gewesen, ein „Radical-Democrat“3 und politischer Journalist mit gefährlich spitzer Feder und großem Gehör vor allem bei der Lehrerschaft des ganzen mecklenburgischen Landes. Sein Weiterwirken in der christlichen Stadtschule schien beiden Seiten undenkbar, wo doch andere seiner Mitstreiter von 1848 gar des Landes verwiesen worden waren. Der soeben als Grimm-Kritiker bekanntgewordene Sanders war plötzlich arbeitslos.

Kein Zufall war, daß Sanders' „kritische Beleuchtung“ des Deutschen Wörterbuchs (DWB) so viele konstruktive Elemente enthielt, daß der bekannte Leipziger Verleger J. J. Weber ihm anbot, mit einem eigenen deutschen Wörterbuch in Konkurrenz zum DWB zu treten. So wurde der Lehrer zum Wörterbuchschreiber und Privatgelehrten in Sachen deutscher Gegenwartssprache. Beruflich und sozial geriet er damit in ein folgenreiches Abseits.

Durch Herkunft und Familie, insbesondere durch die Hausgemeinschaft mit seinem Bruder, dem Lederhändler Alexander Sanders, war Daniel dem mittleren Wirtschaftsbürgertum verbunden, aber er selbst mußte seine eigene Familie, Frau und Kind, vom väterlichen Erbteil und von mühsam erkämpften Honoraren unterhalten. Die Bücher des Pädagogen und Sprachforschers wandten sich vor allem an das Bildungsbürgertum, dem Sanders selbst nur zu gern angehört hätte - als Lehrer oder Universitätsdozent oder vielleicht auch als Bibliothekar. Doch Beamtenberufe und Berufe mit staatlicher Zulassung waren Juden

3 Selbstbezeichnung in der von Sanders redigierten Zeitschrift Blätter für freies Volksthum, Neustrelitz 1848/49. 
fest verschlossen. Ausnahmen bestätigen die Regel, daß dies nämlich nur per Gnadenakt, nicht aus Recht geschehen konnte. Wissenschaftssoziologisch war Sanders' Un-Position insofern gleich dreifach begründet:

1.) Als nicht verbeamtungsfähiger Lehrer galt er der institutionellen Wissenschaft in Universitäten und Akademien nicht als ebenbürtiger Diskussionspartner.

2.) Er war ausdrücklich in erster Linie an der deutschen Gegenwartssprache und weniger an ihren älteren Stufen interessiert und vertrat damit eine noch nicht etablierte und akzeptierte Disziplin: die Neuphilologie Deutsch. Für Sanders' Forschungsgegenstand gab es überhaupt noch keine Mitinteressenten und kaum ein Diskussionsforum.

3.) Sanders machte vor allem aus seiner rationalistisch begründeten Opposition zur historisch-etymologischen Methode der Grimm-Germanistik keinen Hehl, und er verbrämte seine Kritk an ihren antiaufklärerischen Tendenzen nicht aus Ehrfurcht vor den Gründervätern, so daß er persönlich in Ungnade fiel und sich zeitlebens Angriffen ausgesetzt sah, die ihn moralisch, als Charakter herabzusetzen versuchten. Auch daß er Jude war, kam bis in unser Jahrhundert einigen Angreifern sehr gelegen.

Die Brüder Grimm und ihre unmittelbaren Schüler und Nachfolger haben Sanders und seine Bücher nach Kräften ignoriert und - sobald das nicht mehr ging - bekämpft. Gleichwohl hat Jacob Grimm sich in einem seiner wichtigsten Texte in beträchtlichem Maße von diesem Kritiker beeinflussen lassen. Seine Wörterbuchvorrede von 1854 gilt bis heute als der programmatische Text der germanistischen Lexikographie im 19. Jahrhundert und wird immer wieder auch als Quelle für Untersuchungen zur Sprachtheorie Jacob Grimms herangezogen; liest man sie aber aus anderer Perspektive, nämlich vom wissenschaftssoziologischen und publizistischen Kontext her und speziell unter Berücksichtigung von Sanders' Kritik, enthüllt sich ihr weitgehend apologetischer Charakter (s. u.).

Das immense Belegmaterial, das Sanders zur Erarbeitung seines Wörterbuchs der deutschen Sprache angehäuft hatte ${ }^{4}$, bildete den Grundstock für weitere ein- bis zweibändige Wörterbücher. Sanders sah sich nicht nur innerhalb der deutschen, sondern der gesamteuropäischen Lexikographietraditionen nach Anregungen zur Bereicherung der deutschen Wörterbuchlandschaft um. Aber ganz gleich, ob er sich auf Heyses Fremdwörterbuch, Eberhards distinktive oder P. M. Rogets kumulative Synonymik bezog - die Auseinandersetzung mit dem jeweiligen Vorbild führte immer zu der für Sanders typischen Verknüpfung von Kritik und Innovation. Die Kritik entsprang dabei einmal dem Bewußtsein, daß sich die Situation von Gesellschaft und Sprache seit Mitte des 19. Jahrhunderts grundlegend verändert hatte; zum zweiten entsprang sie sei- 
nen methodischen Ansprüchen an eine sowohl gegenstands- als auch adressatengerechte Darstellungsform. Die Vorwegnahme der Fragen von Wörterbuchbenutzern, die keine akademisch-philologische Qualifikation besaßen, besaß in Sanders' Wörterbüchern einen hohen Stellenwert, der sich bis in sprachtheoretisch-konzeptionelle Entscheidungen hinein auswirkte. Er wandte sich nicht primär an das sozial und bildungspolitisch dominierende Bildungsbürgertum, das ein herausragendes Interesse an einer auch sprachhistorischen Sicherung nationaler Identität besaß; diese Adressatenschicht wurde in erneuter Eingrenzung auf philologisch-wissenschaftlich interessierte Leser, de facto für das Grimmsche Wörterbuch bestimmend. Sondern Sanders wandte sich an den gröBeren, aber bildungsgeschichtlich weniger einflußreichen bzw. weniger erforschten Teil des Bürgertums, der seinen ,soziologischen Ort in den Tätigkeitsfeldern außerhalb der bildungsbürgerlichen Lebensbereiche [...] in den Tätigkeits- und Anwendungsfeldern der Naturwissenschaft und Technik, im Handel und im Gewerbe" 5 hatte. Ihr Nationalbewußtsein hielt immer noch Verbindung mit dem Patriotismusideal der Aufklärungszeit und war internationalem Austausch von Waren und kulturellen Inhalten gegenüber offen. Mit Reichmann (1990) wird diese zweite, neben der literaturnahen existierende Sprachgebrauchstradition auch in ihren Auswirkungen bis heute unterschätzt. Sanders' sprachpädagogisches Konzept insgesamt bestätigt nicht nur die Existenz dieser Tradition, sondern auch den Bedarf der sie tragenden sozialen Schicht an Hilfen bei Problemen sprachlicher Kommunikation.

Das gemeinsame Motiv seiner lexikographischen und übrigen sprachdidaktischen Werke (Deutsche Sprachbriefe, Stilmusterbuch, Wörterbuch der Hauptschwierigkeiten in der deutschen Sprache, u. a.) war die Konsolidierung derjenigen Varietät, die die in seinen Augen politisch führungsberechtigte Schicht aus Bildungs- und Wirtschaftsbürgertum zur Bewältigung ihrer kommunikativen Aufgaben benötigte. Was Sanders den ,tatsächlichen Sprachgebrauch der Gegenwart" nannte, umfaßte nicht nur die Sprache der deutschen Klassik, vor allem Goethes und Lessings, sondern vor allem die Ausdrucksmittel von Handel, Verkehr, Gewerbe, Militär und Verwaltung. ${ }^{6}$ Leitlinie seiner Sprachauffassung war nicht literarische Qualität oder muttersprachliche Unverdorbenheit, sondern Klarheit und Unmißverständlichkeit.

Die politische Einigung von 1871 hatte für Aktivitäten und Interessen dieser wirtschaftsbürgerlichen Schicht rechtliche und wirtschaftliche Hindernisse beseitigt; ihr wollte Sanders das Bewußtsein der sprachlichen Einheit an die Seite stellen, indem er regionale Varianten ausgrenzte und funktionale Varianten als solche kenntlich und damit kommunizierbar machte.

Im Ziel der sprachlichen Einheit scheint Sanders zumindest rhetorisch mit dem kulturellen Nationalismus des späten 19. Jahrhunderts konform zu gehen;

5 Reichmann 1990, 233.

6 Der Kommunikationsbereich Industriearbeit ist bei Sanders noch sehr schwach vertreten. 
doch die enge Verknüpfung mit dem Ziel, zur Sicherheit im Gebrauch der Muttersprache zu verhelfen, schließt jede national motivierte Hypostasierung der Sprache aus. Sanders' kulturellem Patriotismus fehlen jegliche aggressiven fremdenfeindlichen Elemente; dies zeigt vor allem seine Einstellung gegenüber Fremdwörtern. Im Gegenteil, da Sprache für ihn im wesentlichen Kommunikationsinstrument ist, stellt sie das Medium internationaler Kontakte dar. Sanders' Vorstellungen von der deutschen Kultur leitet sich von der Aufklärung her, von der Idee der Bildung des Individuums und der einzelnen Völker zur Humanität. Nicht zufällig wurzelt für ihn, den jüdischen „Germanisten“7, deutsche Sprache und Kultur nicht im germanischen Mythos, sondern in der Trias der jüdischen, griechischen und römischen Antike. Und mit der Antike steht das Europa der Gegenwart in einem gewissen Kontinuitätszusammenhang. Dies ist der kulturelle Rahmen, in dem Sanders seine Auffassung von Sprachbildung ansiedelt. Sein Ziel der Befähigung zum sicheren Gebrauch der Muttersprache setzt für ihn eine rationale Sprachauffassung voraus. Rational bedeutet nicht rationalistisch im Sinne einer Unterwerfung der Sprache unter die menschliche Vernunft, sondern gibt die Haltung an, mit der sich der Sprachteilhaber der Sprache und der ihr eigenen Struktur nähert: Es ging für ihn darum, die Vielfalt, Flexibilität und Situationsangemessenheit der Ausdrucksmittel zu erkennen bzw. zu vermitteln.

Wir haben es bei Sanders mit einer Sprachaufklärung zu tun, die sich in wesentlichen Aspekten von derjenigen der Repräsentanten Adelung und Gottsched unterscheidet; er ist rational, aber kein Vernunftsprachler; patriotisch, aber kein Purist; und er will Orientierung in Normenunsicherheiten vermitteln, erkennt aber keine singulären Sprachrichter an.

Wie hätte eine Germanistik ausgesehen, die dieses Konzept aufklärender und zugleich nationalbewußter Sprachreflexion schon im 19. Jahrhundert in ihr wissenschaftliches und methodisches Programm aufgenommen hätte? Und wohin hätte sie sich entwickelt? Wir wissen, daß die Opposition der Romantik gegen „die Aufklärung“" so stark war, daß eine noch so eingegrenzte Synthese von ihrer Seite aus nicht in Frage kam. Die historisch-etymologische Germanistik hat die durch Rationalismus, Normativität (und z. T. auch: Purismus) zu undifferenziert charakterisierte Aufklärung abgelehnt und dabei vielleicht übersehen, da $B$ es in dieser großen geistigen Bewegung auch noch andere Sprachauffassungen gab. Schriftsteller wie Lessing, J. H. Voß, J. J. Engel und $\mathrm{K}$. Ph. Moritz haben mehr durch praktisches Vorbild als durch explizit sprachtheoretische Äußerungen die Auffassung vermittelt, die Sanders lexikographisch umsetzte.

Sanders' Fall zeigt, daß die Aufklärung, auch die sprachbezogene, im 19. Jahrhundert nicht überholt, überwunden und tot war; natürlich war sie im

7 Er hat sich selber nie so bezeichnet, wohl um sich nicht in die Nähe der historischen Schule und ihres Ansatzes zu rücken. 
Hinblick auf die neue sprachgeschichtliche Situation einer Gesellschaft zu modifizieren, die wir heute als Industrialismus kennzeichnen, die Sanders aber offenbar primär in politisch-rechtlicher und wirtschaftlicher Hinsicht verändert erlebte. Bis 1869 Schutzjude des Großherzogs von Mecklenburg-Strelitz schrieb er sein Wörterbuch in der Hoffnung, endlich die Früchte der Aufklärung in Form rechtlicher Gleichstellung und gesellschaftlicher Anerkennung ernten zu dürfen.

\section{Probleme der Historiographie der Germanistik}

Die Historiographie der germanistischen Sprachwissenschaft hat mit Modifikationen der Sprachaufklärung und mit ernstzunehmenden Alternativen zum dominierenden Paradigma der historischen Schule in diesem Zeitraum nicht gerechnet. Die Gründe liegen 1.) in der einschränkenden Bestimmung der Art oder des Niveaus von Sprachreflexion, die als Gegenstand der Fachgeschichte überhaupt in Betracht zu kommen schien, 2.) in der primär institutionell-sozialen Fixierung des Wissenschaftlichkeitsbegriffs von Jacob Grimm bis heute, und 3.) im Geschichtsbild, das die Germanistik hinsichtlich ihrer Anfänge im 19. Jahrhundert hegt.

Obwohl in vielen Arbeiten die methodischen Schwierigkeiten der Fachgeschichtsschreibung durchaus reflektiert werden ${ }^{8}$, wirken sie sich im Hinblick auf die Germanistikgeschichte immer noch verzerrend aus.

\subsection{Die Bestimmung des Gegenstands}

Sprachwissenschaftliche Fachgeschichten, gleich ob synchron oder diachron ich beziehe mich im folgenden auf diejenige der sprachwissenschaftlichen Germanistik im 19. Jahrhundert —, setzen sich einen bestimmten Ausschnitt aller sprachreflexiven Äußerungen einer Sprachgemeinschaft zum Gegenstand. Dieser Ausschnitt kann räumlich, zeitlich, sozial, thematisch und quellenbezogen näher bestimmt werden. Das relativ größte Gewicht und der größte Begründungsaufwand wird in den Gegenstandsbestimmungen der Zeitdimension zugestanden. Wissenschaftsgeschichten stützen sich dabei auf interne Daten (die erste germanistische Professur: 1810; Jacob Grimms Deutsche Grammatik: 1819) und politische Zäsuren (1848 und 1871). Demgegenüber ist man in der Sprachgeschichte inzwischen zu der Überzeugung gelangt, daß Periodisierung und Epochenbenennungen nach der Gegenwart hin zunehmend sozialgeschichtlich ausgerichtet sein müssen. ${ }^{9}$

Vgl. die einleitenden Überlegungen von Monreal-Wickert 1977.

9 Begründung und Überblick über die seit 1950 geführte Diskussion von Prinzipien und Praxis der Periodisierungen bei von Polenz 1989. Vgl. G. Wolff 1986, 180ff und Eggers 1977, $121 \mathrm{ff}$. 
Die soziale Dimension, d. h. die Unterscheidung zwischen akademisch institutionalisierter und öffentlicher, nicht fest organisierter Sprachreflexion, wird in den meisten Fachgeschichten als solche nicht reflektiert und begründet, sondern unproblematisiert vorausgesetzt, wie es die räumliche Dimension (das Verbreitungsgebiet des Deutschen) tatsächlich ist. Da die Geschichtsschreibung grundsätzlich auf schriftliche Quellen und kaum auf (noch) lebende Personen angewiesen ist, muß sich die Gegenstandsbestimmung als Kriterienkatalog für die Auswahl der relevanten Quellen nutzbar machen lassen.

Tatsächlich folgen die meisten germanistikgeschichtlichen Darstellungen mehr oder weniger unausgesprochen einer sozialen, genauer institutionellen Bestimmung ihres Gegenstands: Das primäre Interesse gilt dem theoretischen Gehalt derjenigen Texte, die von Lehrstuhlinhabern und/oder Akademiemitgliedern für die gruppeninterne Kommunikation verfaßt worden sind.

Für die Historiographie der germanistischen Anfänge ist dies insofern problematisch, als eine entwicklungsgeschichtliche und vergleichende Perspektive im Hinblick auf die Zeit vor 1800 damit gar nicht möglich ist. Das Kriterium, nur in institutioneller Bindung entstandene Sprachreflexion sei Sprachwissenschaft und damit von historiographischem Interesse, ist auf die dem 19. vorangehenden Jahrhunderte gar nicht anwendbar, wenn man an der Identität des Gegenstands Sprachwissenschaft über die Zeitgrenze hinweg festhalten will, die durch die Einrichtung der ersten germanistischen Professuren seit 1810 markiert wird. Obwohl ein mehr oder weniger explizit institutionell begründeter Begriff von Wissenschaft in fast allen Darstellungen dominiert, wird in den Einleitungen oft eine Linie von der neuzeitlichen oder „modernen“ Sprachwissenschaft bis zurück zu „Sprachbetrachtungen“ antiker Völker gezogen (vgl. Schmitter 1982, S.80ff), die Jahrhunderte oder gar Jahrtausende vor unserer Zeitrechnung liegen. In Darstellungen, die einen Zeitraum behandeln, in dem das 19. Jahrhundert mit älteren zusammengefaßt ist, wie etwa bei Arens (1974), wird schon „die bloße Beschäftigung mit einem Sprachproblem“ als Aufnahmekriterium gewertet (Schmitter 1982, 84). Daß beispielsweise das Werk von Joh. Chr. Adelung in die Geschichte der germanistischen Sprachwissenschaft hineingehört, hat wohl noch niemand infrage gestellt, obwohl dieser Sprachforscher von seiner eigenen Bildungsgeschichte und von seiner Position im Bildungssystem seiner Gesellschaft her ebenso außerhalb von Universität und Akademie stand wie viele unbeachtete Sprachforscher nach ihm (vgl. Bahner 1984b).

\subsection{Die Bestimmung von Wissenschaftlichkeit}

Die Bestimmung der akademisch institutionalisierten Sprachreflexion als nahezu einzigen Gegenstand der Historiographie beruht auf einem bestimmten, aber nur selten explizit gemachten Maßstab von Wissenschaftlichkeit. Das zentrale Kriterium, die Wissenschaftlichkeit sprachreflexiver Texte und damit ihre Dignität als Gegenstand der Fachgeschichte zu bestimmen, ist dabei das Kriterium der Methodizität der Beschäftigung mit Sprache. Es gewinnt desto mehr 
an Einfluß, je näher der Gegenstandsbereich zeitlich an die Gegenwart heranrückt (vgl. Schmitter 1982, 86f), und ist somit ebenfalls für den Bruch verantwortlich, mit der die Sprachwissenschaft vor 1810/1819 in vielen Darstellungen zur Prähistorie gemacht wird. Insbesondere wird ein hoher Explizitheitsgrad der Methodik mit Wissenschaftlichkeit gleichgesetzt (Schmitter 1982, 89). So wird der letztlich immer von heute aus gedachte methodische Standard historiographisch bestätigt. ${ }^{10}$

Auch das Kriterium der Methodizität führt zu gewissen Widersprüchen. Erstens gehörte es zum Programm der sprachwissenschaftlichen Romantik, Methodenregeln - weil zu einengend - abzulehnen, da sie als Charakteristikum des für überwunden gehaltenen aufgeklärt-rationalistischen Paradigmas galten. Zweitens ist methodisch geregelte Sprachreflexion auch außerhalb der akademischen Institutionen nachzuweisen, vor allem im Bereich der Sprachdidaktik, der aber gerade nicht dem Gegenstandsbereich der Fachgeschichte zugeschlagen wird.

Ein ausdrückliches Methodeninteresse kann unterschiedlichen Zielen folgen: der Entwicklung theoretischer Modelle, den empirischen Verfahren bei Auswahl und Interpretation sprachlicher Materialien oder dem Erklärungs- und Vermittlungsaspekt der theoretisch oder empirisch gewonnenen Ergebnisse. Vesper (1980) hat deshalb vorgeschlagen, vor allem im Hinblick auf das 19. Jahrhundert zwischen Sprachtheorie und Sprachbeschreibungstheorie zu differenzieren, die gleichermaßen dem Maßstab der Methodizität genügen. Beispielsweise ist die Frage nach Elementen und Struktur der Grammatik des Deutschen (,Was ist eine deutsche Grammatik und wie ist sie aufgebaut?“) wissenschaftlich und methodisch genauso anspruchsvoll wie die Frage nach den Darstellungsmöglichkeiten dieser Struktur (,Wie schreibt man eine Grammatik des Deutschen?").

Grammatikographen und Lexikographen haben sich immer schon mit modelltheoretischen, empirischen und interpretatorischen Entscheidungen befassen müssen, die für Sprachtheoretiker eher sekundäre Bedeutung hatten. Zum Beispiel die lexikographische Entscheidung über das Verhältnis von Gesamtbedeutung und Einzelbedeutung. Der institutionell bestimmte und methodisch legitimierte Begriff von Wissenschaftlichkeit geht unmittelbar zurück auf den Wissenschaftlichkeitsbegriff der Universitätsgermanisten, insbesondere auf die Auffassung Jacob Grimms.

Im Zusammenhang mit seinem bekannten Verdikt des Grammatikunterrichts in der Muttersprache ${ }^{11}$ formulierte Jacob Grimm in der Einleitung zur Deut-

10 Nach Schmitter $(1982,90)$ trennt allein Coseriu scharf zwischen Wissenschaftlichkeit und Methodik.

11 „musz ich gleich vorweg erklären, warum ich die art und den begriff deutscher sprachlehren, zumal der in dem letzten halben jahrhundert bekannt gemachten und gutgeheiszenen für verwerflich, ja für thöricht halte. man pflegt allmälig in allen schulen aus diesen werken unterricht zu ertheilen und sie selbst erwachsenen zur bil- 
schen Grammatik (1819) und später erneut in einer Akademierede ${ }^{12}$ seinen Begriff von Wissenschaft. Ivo hat herausgearbeitet, wie mit dem Verdikt geradezu das Signal gesetzt wurde zur „Abwendung von einem Verständnis der Wissenschaft als auf praktische Zwecke unmittelbar bezogen [...] hin zu einer [Sprachwissenschaft], die, von praktischen Rücksichten frei, nur darauf zielt, neue wissenschaftliche Gesetze [zu] entfalten“" (Ivo 1990, 589). ${ }^{13}$

Der von Grimm beabsichtigte Bruch mit der Tradition des Rationalismus und der Aufklärung mit ihrem Nützlichkeitsdenken beinhaltet ,wissenschaftstheoretische(n) Bestimmungen, die den Wissenserwerb innerhalb der Institution ,Wissenschaft " von Zwecksetzungen anderer Institutionen abkoppeln" (Ivo 1990, 590). Mit der Gleichsetzung von , anwendungsbezogen' und ,unwissenschaftlich" durch Jacob Grimm begann die Tradition eines Maßstabs von Wissenschaftlichkeit, in der sprachpädagogische Fragen, bildungstheoretisch bedeutsame Fragen des Schriftspracherwerbs, die Erforschung der Standardsprache auf sämtlichen Ebenen und ihres Verhältnisses zu regionalen, sozialen, funktionalen Varietäten und auch Fragen nach der „Rolle nationalsprachlicher Konzepte“ bzw. nach der „Rolle der Sprache in der Bildungsgeschichte der Menschheit" aus dem Blick gerieten. (Ivo 1990, 590, 592).

Die strikte Grenzziehung zwischen Wissenschaft und Nicht-Wissenschaft, wie sie von Seiten der Fachgermanistik vorgenommen wurde, war im 19. Jahrhundert kein gesamtgesellschaftlicher Konsens. Meyer's Konversationslexikon von 1867 (2. Auflage) bestimmte Wissenschaft unabhängig von jeder institutionellen Beschränkung und mit auffallendem Hinweis auf Anwendbarkeit und Praxisbezug.

Die Intentionen jenes nicht auf die akademischen Institutionen beschränkten Verständnisses von Sprachwissenschaft sieht Neumann (1988) im politischideologischen Zusammenhang einer gesellschaflich erwünschten „Effektivierung der sprachlichen Kommunikation“" in den Jahrzehnten zunehmender Indu-

dung und entwicklung ihrer sprachfertigkeit anzurathen [...] den geheimen schaden, den dieser unterricht, wie alles überflüssige, nach sich zieht, wird eine genauere prüfung bald gewahr." Jacob Grimm, Vorrede zur 1. Auflage der Deutschen Grammatik, in: Grimm 1890, 30.

12 Über Schule Universität Academie, in: Neumann/Schmidt 1984, 212-249.

13 Auch im persönlichen Umgang wirkte Jacob Grimms Gelehrsamkeit exklusiv, sogar dort, wo er sich in einem politischen und nicht wissenschaftlichen Kontext äußerte, wie in der Paulskirche. Vilmar schrieb am 31.5.1849 an Weigand:

Jakob Grimms Existenz in der National-Versammlung ist unsern Studien oder vielmehr deren Geltung nicht förderlich gewesen. Doch darnach fragen wieder die Grimms nicht - sie wollen ihre Sachen eben nicht aus dem Kreise der Gelehrsamkeit gerückt sehen; exklusiv oder gar nicht! ist ihr alter, wenn auch unausgesprochener Wahlspruch. (Zit. Janota 1980, 41).

Daß Jacob Grimm nicht gern lehrte, sich nicht gern auf Ansprüche und Bedürfnisse von Adressaten, seien es Studenten in seinen Vorlesungen oder Benutzer des Wörterbuchs, einlieB und für sich selbst keinerlei Gewinn daraus schöpfte, ist in der Forschungsliteratur über Grimm bekannt und belegt (z. B. bei Ebel 1984, 62ff). 
strialisierung, Internationalisierung des Handels und des Kolonialismus. Er stellt fest, daß „sich die Spitzen der neuentstehenden Wissenschaftshierarchie diesem Bedürfnis noch entzogen“ (Neumann 1988, 12). Als Gegenspieler-Institutionen zu Universität und Akademie, die geschaffen wurden, um dem gesellschaftlichen Bedürfnis Genüge zu tun, nennt Neumann Sprachgesellschaften und Vereine; die nicht kleine Gruppe der Gymnasiallehrer, die sprachwissenschaftlich gearbeitet haben (vgl. Knobloch 1986, 176), ist noch hinzuzufügen.

\subsection{Das Geschichtsbild}

Gegenstandsbestimmung und Wissenschaftlichkeitsbegriff haben quer durch die ideologischen Lager die Geschichte der Germanistik als autochthone Ideenund Theoriendynamik gezeichnet. An den einzelnen wissenschaftsgeschichtlichen Modellen ist besonders aufschlußreich, wie sie Bruch oder Kontinuität zwischen den Anfängen der Germanistik und den sprachreflexiven Konzeptionen der Aufklärung darstellen.

Bis Bahner (in: Bahner/Neumann 1985) auf die Gleichzeitigkeit von Kontinuität und Diskontinuität auch dieser Epochen aufmerksam machte, beherrschte mehr oder weniger die Vorstellung von einer Art Urknall zu Beginn des 19. Jahrhunderts das Bild.

Nach dem Standardwerk von Helbig (1973), der das 19. Jahrhundert im ersten Kapitel als Vorgeschichte de Saussures behandelt, brach die Sprachauffassung der Aufklärungszeit einfach ab, als die „Namen“ Bopp, Rask und Grimm der deutschen Sprachwissenschaft erstmals „eine gewisse Geltung“ verschafften, die demnach vorher trotz der Namen Adelung, Campe, Gottsched u. a. gefehlt hatte. Davon abgesehen, daß Bewertungen solcher Art die Frage aufwerfen, wer sie wann und nach welchem Maßstab wissenschaftlicher Geltung vorgenommen hat, zeigt die Art, wie Helbig die Sprachauffassung von vor 1800 charakterisiert, was hier als der Wissenschaftlichkeit entgegengesetzt angesehen wird: Orientierung an der Praxis, d. h. am Gebrauch von Sprache durch Sprecher, und Orientierung an Modellen aus der Logik, die Helbig (ebenda 21) an anderer Stelle ,logizistisch“ nennt.

Es sind immer wieder drei Epitheta, mit denen die deutsche Sprachaufklärung in der Fachgeschichte charakterisiert wird: rationalistisch (Formulierungsvarianten: logisch, systematisch, philosophisch), normativ (Varianten: autoritativ, trocken, pedantisch) und häufig auch puristisch. Die Wertigkeit dieser Prädikate ist heute noch die gleiche wie bei Jacob Grimm. Damals wie heute dient die Abwertung der Sprachauffassung der Aufklärung dazu, den Traditionsbruch bzw. den Innovationsschub durch die historisch-vergleichende Sprachwissenschaft zu Beginn des 19. Jahrhunderts zu betonen bzw. zur Grundlage des Wissenschaftsgeschichtsbilds zu machen. Dies sei verdeutlicht an der Darstellung Adelungs bei Gipper/Schmitter (1985, $41 \mathrm{ff})$.

Adelungs Gesamtwerk wird nach Kategorien aufgeteilt, die dem neuen Paradigma der historisch-vergleichenden Sprachwissenschaft entnommen sind. 
Adelungs komparatistische Leistung wird insgesamt eher negativ beurteilt und zwar als „eine eigenartig zwielichtige Stellung [einnehmend]“ (ebenda 41), „widersprüchlich“, ,sehr merkwürdig“" und als rückschrittlich (ebenda 42). Das zweite Adelungsche Fragment, „Ansätze“ (ebenda 52) zu einer historischen Grammatik, wird ausschließlich von Jacob Grimm her dargestellt, $d$. h. aus der Perspektive seines mächtigsten Kritikers, der im Kontrast zur logisch-philosophischen Auffassung seines Vorgängers Adelung das Innovative seiner historischen Auffassung umso deutlicher hervortreten lassen möchte. Wissenschaftshistoriker von heute machen sich das 160 Jahre alte Urteil dieses Kritikers über Adelung im Wesentlichen zu eigen: Grimm habe sich ,zu Recht“ von den Büchern Adelungs distanziert (ebenda 56). Ferner wird Adelung vorgeworfen, daß er „seine eigenen Grundsätze nicht befolgt und stattdessen zwei normative Grammatiken geschrieben" habe (ebenda 57). Das Stigmatisierende des Epithetons , normativ ' gilt für die Linguistik der 70er und 80er Jahre des 20. Jahrhunderts, und es galt auch für Jacob Grimm - zufällig, könnte man sagen, denn die Motive, die zur Ablehnung des Normeninteresses führen, sind in beiden Fällen grundverschieden.

Aus der Identifizierung sehr alter und sehr neuer Urteile über Adelung resultiert die historiographische Deutung des Prozesses, da $B$ nämlich Adelung und die gesamte Sprachbetrachtung der Aufklärung keine wissenschaftlich relevante Nachwirkung im 19. Jahrhundert mehr gehabt zu haben scheinen, als ein Kausalzusammenhang von Lohn aufgrund besserer Leistung: „Infolgeddessen blieb es [...] Grimm vorbehalten, [...] eine neue Disziplin zu etablieren" (ebenda 57). Auch Adelungs lexikographische Leistung wird ausschließlich aus der bekannten abwertenden Perspektive Grimms dargestellt. Die Wissenschaftshistoriker stützen sich hier sogar auf den Wortlaut einer Kritik, die Grimm öffentlich an Adelungs Wörterbuch vornahm, als er 1838 für das eigene Unternehmen zu werben begann (Gipper/Schmitter 1985, 58).

Ein weiteres, bei der Darstellung der Fachgeschichte in der 2. Jahrhunderthälfte verbreitetes Modell möchte ich das Staffellauf-Modell nennen. Hier wird die Linie von den „Männern“ der Romantik geradewegs „zu den sog. Junggrammatikern und zu einer neuen Epoche positivistischer Sprachbetrachtung mit naturwissenschaftlichem Einschlag" geführt (Gipper/Schmitter 1985, 16), gerade so als hätten die Romantiker die Staffette ihrer Theorien und Methoden unmittelbar an die Junggrammatiker weitergereicht bzw. diese sie aus der Hand jener entgegengenommen. Auch Helbig $(1973,14)$ konzeptioniert diesen Teil der Sprachgeschichte als einen einlinigen Wechsel der Auffassungen: „die romantische Sprachphilosophie [...] räumt der nächsten, naturwissenschaftlich und positivistisch ausgerichteten Generation den Platz."14 senschaft und damit eher die Ablösung als die Fortführung der romantischen Tradition. 
Die Metapher der „Generation“15 spielt überhaupt eine wichtige historiographische Rolle. Mit ihr wird unterstrichen, daß hier das Bild von einer Kette aus Tradition-Rezeption-Innovation, vom Weiterreichen und Fortentwickeln der Methoden und Theorien auf der sozialen Insel der scientifc community, vom Auswechseln der Paradigmen ${ }^{16}$ ohne Alternativen und ohne Brüche, die quer zur historiographisch gezogenen Linie liegen könnten, vorherrscht.

Das Modell des Staffel-Laufs der wissenschaftlichen Generationen verführt zu einem zumindest schiefen Bild vom Verhältnis alter und neuer Konzeptionen sprachreflexiven Handelns im 19. Jahrhundert. ${ }^{17}$

Einen ersten Versuch, Fachgeschichte um eine soziale oder politische Dimension zu erweitern, unternahmen einige „ideologiekritische“ Arbeiten seit Anfang der 70er Jahre. ${ }^{18}$ Während Müller $(1974,1)$ seinen Ansatz als ,wissenschaftssoziologisch“ bezeichnet und ihn sowohl von „Hagiographien irgendwelcher Wissenschafts-,Väter““ als auch von der „,eigendynamischen Methoden- und Geistesgeschichte " absetzt, aber dennoch den Gegenstand seiner Untersuchung institutionell auf „die Germanistik der Phase 1806-1848“ beschränkt, versucht Janota (1980), „die Grundzüge der Germanistikgeschichte von der politischen Geschichte her" zu schreiben. Es gelingt ihm weitgehend, drei durch Zäsuren von 1848 und 1871 gebildete Perioden auf die interne Entwicklung des Faches und seine Außenwirkung abzubilden. Janotas Modell läßt besonders die Unterschiedlichkeit des Nationalitätsgedankens deutlich hervortreten, wie er einmal vor 1848 als „Patriotismus“ und einmal nach 1871 als „Nationalismus“ auch in der Germanistik geäußert und in das Selbstverständnis des Faches eingeordnet worden ist.

Die historisch-vergleichende Methode als das dominante Paradigma des Fachs wandelte sich zwischen Jahrhundertbeginn und ca. 1850 in einer Weise, die in Janotas Modell als allmählicher Verlust der durch Rekurs auf die Ge-

15 Zwischen den Geburtsdaten der Romantiker und der Junggrammatiker liegen allerdings mehr als jene 25 Jahre, die für eine Generation in nicht-metaphorischer Verwendung angesetzt werden. Nach Putschke (ebenda) ist der Beginn der junggrammatischen Schule auf 1876 zu datieren.

16 Der Ausdruck Paradigma wird in dieser Arbeit so verwendet, wie er in der allgemeinen Wissenschaftssprache inzwischen monosemiert wurde (vgl. Artikel Paradigma von Th. Rentsch in: Historisches Wörterbuch der Philosophie, Bd. 7, Basel 1989, 74-81); die von seinem Erfinder Thomas S. Kuhn später begründete Ersetzung durch den angemesseneren Ausdruck wissenschaftliche Matrix ist in der Anwendung auf mein Thema zu unhandlich und leicht mißverständlich.

17 Allerdings ist unbestritten, daß einiges für das einlinige historische Modell vom „Staffel-Lauf“" spricht. Ein solches Modell scheint in der Person Scherers geradezu Gestalt zu gewinnen. Neumann $(1988,5)$ lokalisiert den „Kampf zwischen der von seinen Lehrern übernommenen romantischen Tradition und der selbstgewählten Konversion zum Positivismus“ in Scherers eigenem Inneren. Auch Zarnckes persönliche Beziehung zu einigen Junggrammatikern scheint das Staffetten-Modell nahegelegt zu haben (Neumann 1988, 10).

18 Z. B. Emmerich 1971, Engster 1986, Greß 1971, Janota 1980, Müller 1974. 
schichte gewonnenen ,handlungsleitende(n) Perspektive(n) zur Überwindung politischer Mißstände“ (Janota 1980, 21) und als zunehmende „Fluchtbewegung aus der bedrückenden Gegenwart“" (ebenda 22; ähnlich Greß 1971) gedeutet wird. Mir scheint, daß die degenerative Deutung der Fachgeschichte als Verlust, Flucht etc. zu sehr dem Identifikationsbedürfnis der 70er Jahre mit den freiheitlich-emanzipativen Momenten am Beginn der Fachgeschichte verhaftet ist. ${ }^{19}$ Janota selbst warnt mehrfach vor einer die Anfänge mythisierenden Neuorientierung der Germanistik (z. B. ebenda 27f) - eine Gefahr, die allerdings erst durch sein Geschichtsmodell entsteht, in dem mittels Verben wie abgleiten, umschlagen und entarten (alle ebenda 31) der Anfang eines gesetzten Prozesses aufgewertet und hypostasiert wird.

Die nach Jacob Grimms Tod entstehenden Mythisierungen in den Selbstdeutungen des Fachs und seiner Anfänge können in Janotas Modell wie auch im oben angeführten Staffetten-Modell kaum systematisch einbezogen werden. Die selbstreflexiven Faktoren der Entwicklung sind in beiden Modellen höchstens als Relikte überwundenener Positionen $\mathrm{zu}$ fassen. Am ausführlichsten werden sie von Wyss $(1979,1-53)$ thematisiert, bei dem aber wiederum die politik- und sozialgeschichtliche Einbettung der Fachgeschichte fehlt.

Die entscheidende Schwäche der von Janota und Müller herangezogenen Modelle der historisch-politischen Periodisierung liegt wiederum in dem sehr engen Gegenstandsbereich. Sie beschränken sich auf die Lehrstuhl-Germanistik, d. h. auf die Ordinarien, seit F. H. von der Hagen die erste Professur für deutsche Sprache und Literatur überhaupt an der 1810 neugegründeten Berliner Universität erhielt. Die Tatsache, daß sich die Germanistik vor allem nach der Reichsgründung 1871 in zunehmendem Maße der Literaturgeschichte zuwandte, wird als „Einengung“ und „Eingrenzung“ (Janota 1980, 37) und als „Philologisierung " negativ bewertet. ${ }^{20}$ Für Janota ist die Philologisierung indirekter Auslöser für eine zeitgleiche Gegenbewegung, die stärkere Popularisierung. In der trotz anderslautender Vorsätze strikt wissenschaftsinternen Perspektive Janotas wird sie negativ als unwissenschaftlich, als „Mißbrauch“ von Forschungsergebnissen bewertet:

Der Schaden, den die Germanistik durch den offenkundigen Erfolg dieses Konzeptes [gemeint sind die populären Literaturgeschichten von Vilmar, Koberstein u. a., U. H.-Z.] erlitt, war weitaus größer als ihre Gefährdung durch mangelndes Publikumsinteresse an einem einseitig philologisierten Fach. (Janota 1980, 41).

19 Janotas ideologiekritische Intention berührt sich mit der von Greß 1971 und mit der Kritik von Emmerich 1971 an der „Volkstumsideologie“.

20 Bezeichnend sind hierfür Formulierungen wie [die literaturgeschichtliche Wende der Germanistik] „schnitt [...] Entwicklungen ab“, „hemmte die Entfaltung“ zur Literaturkritik (Janota 1980, 11); „,beschritt Karl Lachmann einen Weg, der die Germanistik methodologisch in die Arme der klassischen Philologie führte“ (ebenda 35). Zum beispielhaft dargestellten Verhältnis von klassischer Philologie und Linguistik vgl. Jäger 1987. 
Die herablassende Bewertung des Publikumsinteresses verwundert heute, steht sie doch im Widerspruch zu dem Ziel, eine gesellschaftspolitisch bewußte Fachgeschichte zu schreiben.

Die Frage nach dem diachronen Verhältnis der sich zu Beginn des 19. Jahrhunderts konstituierenden germanistischen Sprachwissenschaft und der sich zeitgleich neuorientierenden klassischen Philologie, aus der sich erstere zuvor „emanzipiert“ hatte (vgl. Bahner in Bahner/Neumann 1985, 337f), ist eine sehr wichtige, aber bisher zu selten behandelte Frage. ${ }^{21}$ Am Beispiel Sanders' kann deutlich gemacht werden, daß bei der „Emanzipation“ der Fachgermanistik von der Philologie viel Methodenbewußtsein verloren ging, und daß philologische Traditionen außerhalb der institutionellen Wissenschaft weiterhin fruchtbar waren. Die sog. frühen Semasiologen (vgl. Schmitter 1987), in deren Semantikkonzeption Parallelen zu der von Sanders festgestellt werden können, sind dafür ein weiteres Beispiel.

Jäger 1987 macht sehr deutlich, wie die lange historiographische Abwertung der Philologie und das gegenwärtige Selbstverständnis der germanistischen Linguistik zusammenhängen. Er stellt fest, daß sich letzteres spätestens seit Anfang der 70er Jahre in einer Krise befindet, deren Ursache er in ,ihrer überschwenglichen Entäußerung" an die theoretischen Leitbilder der allgemeinen Wissenschaftstheorie und der allgemeinen Linguistik, also in der Orientierung an einem der romantischen Herkunft des Faches entgegengesetzten Szientismus ausmacht (Jäger 1987, 199): „Die Germanistische Linguistik glaubt sich nur in dem Maße als Wissenschaft konstituieren zu können, wie sie sich von ihrer philologisch hermeneutischen Herkunft löst.“ (ebenda 220). Für die heutige Situation gilt ebenso wie schon für die Junggrammatiker, daß die Identität des Faches, d. h. der institutionell definierten, sich ihm zugehörig fühlenden Gruppe, zu einem ganz wesentlichen Teil auf dem „Modell einer notwendigen Ablösung des Faches von seiner romantischen Herkunft", von - wie Scherer es nannte „romantischer Beschränkung“ und „Einseitigkeit“" beruht (ebenda 200). Das Abgrenzungsargument der neueren Linguistik erweist sich letztlich als Indiz für eine gewisse „Geschichtsblindheit“ (Jäger 1987, 204), für die ,herrschende(n) Verdrängung der philologischen Herkunft des Faches“ (ebenda 205).

In Bahners Geschichtsmodell des mehrsträngigen Prozesses wird die Beschränkung auf den universitär-institutionellen Rahmen von Sprachreflexion aufgehoben, und es kommt die Institutionalisierung als sozialer Prozeß selbst in den Blick. Die sozialgeschichtliche Erweiterung kann die Gleichzeitigkeit von Kontinuität und Diskontinuität beim Übergang von der Aufklärung ins 19. Jahrhundert in weitaus befriedigenderer Weise erklären als eine einlinig auf Theorien beschränkte Wissenschaftsgeschichte.

21 Dies hängt eng mit dem aktuellen Selbstverständnis der germanistischen Linguistik der 80er und 90er Jahre und ihrem gespannten Verhältnis zu Philologie und Hermeneutik (als Methoden) zusammen. 
Einen der zwei Aspekte von Kontinuität in jenem Übergang sieht Bahner darin, daß ,die deutsche Sprache [...] wie andere Einzelsprachen als ein empirisch gegebener Gegenstand begriffen [wurde], der eine autonome Betrachtungsweise erforderte" (Bahner in Bahner/Neumann 1985, 331, vgl. 25). Die Tendenz zur empirischen Grundlegung machte sich schon gegen Ende der Aufklärungsepoche deutlich bemerkbar und setzte sich von der philosophisch-prinzipienhaften $\mathrm{Be}$ trachtungsweise ab. Die historische Betrachtungsweise konnte hier unmittelbar anknüpfen. Ihre Empirie nahm sich lange die Sprache älterer Zeitstufen zum Gegenstand. Erst das nach der Jahrhundertmitte erscheinende Deutsche Wörterbuch der Brüder Grimm ließ in einigen Teilen der Sprachgemeinschaft Erwartungen entstehen, hier werde empirisch der Wortschatz der Gegenwart behandelt oder zumindest in die diachrone Betrachtungsweise einbezogen. An eben diese Erwartungen knüpfte Daniel Sanders an mit seinem Konzept der empirisch-synchronen und zugleich praxisbezogenen Erforschung des Wortschatzes in hauptsächlich semantischer, nicht morphologischer Sicht.

Die Fachhistoriographie ist mit der Annahme einer solchen Komplexität ihres Gegenstands vor eine ungleich schwierigere Aufgabe gestellt als bisher. Es ist aber deutlich, daß eine gewisse Komplexität des Geschichtsmodells notwendig ist, nicht nur, um einen Forscher wie Sanders überhaupt in den Blick zu bekommen, sondern auch um seine Sprachauffassung und seinen methodischen Ansatz historisch einzuordnen.

Die soweit skizzierten Modelle sollten sichtbar machen, wie unterschiedlich Linienführung und Gegenstandsbestimmung in der Historiographie der Sprachwissenschaft sind. Daß dafür ein je anders gelagertes, auf die Gegenwart bezogenes Erkenntnisinteresse verantwortlich ist, sollte nicht bedauert, sondern eher geschichtstheoretisch verstanden werden: Wie Rüsen $(1983,82)$ zeigt, gehört die Bedeutung der erzählten Geschichte für die Lebenspraxis der Adressaten, d. h. der Bezug zu den in der Gegenwartsgesellschaft geltenden Normen und Werten zu den Bedingungen, die erfüllt sein müssen, damit eine historiographische Darstellung als intersubjektiv plausibel oder in gewissem Sinne als wahr gilt. ${ }^{22}$ Die Funktion, die diese Fachhistoriographie für heutige Germanistinnnen und Germanisten hat, liegt eben auch in der Vergewisserung des eigenen sozialen Prestiges in Abgrenzung zu jenen, die sich als Nicht-Wissenschaftler über Sprache äußern.

\subsection{Quellenkritik}

Die germanistische Linguistik ist besser als andere Disziplinen darauf vorbereitet, Status und Wert ihrer historiographischen Quellen zu bestimmen. Historio-

22 Die zwei anderen Bedingungen sind nach Rüsen 1983, 82 die empirische Triftigkeit durch Quellennachweis und der sinnhafte Zusammenhang zwischen Fakten und Normen durch Ideen, die in der Lebenspraxis der Adressaten wirksam sind. 
graphie ist das Resultat eines gezielten Umgangs mit überlieferten Texten, so daß die Gegenstandsbestimmung auch die Form folgender Frage annehmen könnte: „Welche Eigenschaften qualifizieren einen Text in den Augen der Geschichtsschreiber dazu, als sprachwissenschaftlich und damit prinzipiell zur Fachgeschichte zugehörig eingestuft zu werden?“ (Schmitter 1982, 75).

Es bleibt jedoch offen, ob hierbei an die Themen und Inhalte eines möglichen Quellentextes oder auch an dessen kommunikative Funktion gegenüber einer sozial bestimmbaren Adressatengruppe gedacht ist. Von Schmitter (1982, 74) und anderen wird zwar einerseits betont, daß die Geschichte einer Wissenschaft mehr sei als bloße Theoriendynamik, andererseits stehen de facto Theorien und Methoden auch bei der Quelleninterpretation im Vordergrund. Offensichtlich fehlen historiographische Überlegungen, wie der kulturelle, soziale und kommunikative Kontext, in dem theoretische und methodische Äußerungen entstehen, in den Untersuchungsbereich mit einbezogen werden können.

Ein wissenschaftsgeschichtlich oft herangezogener und gedeuteter Text wie die Vorrede Jacob Grimms zum Deutschen Wörterbuch (DWB) von 1852 erfüllte seine kommunikative Funktion gerade nicht primär innerhalb der scientific community, sondern in dem an Bildung interessierten Teil der deutschen Öffentlichkeit. Der Erwartungshorizont der Adressaten setzte sich zusammen aus der spätestens seit Mitte der 30er Jahre des 19. Jahrhunderts öffentlich umlaufenden Idee eines deutschen Wörterbuchs mit nationalrepräsentativer und identitätsstiftender Funktion, aus der Kenntnis der seit 1852 erschienenen Lieferungen des DWB und aus der öffentlichen Diskussion der Frage, ob dieses Wörterbuch die Idee des Nationalwörterbuchs denn tatsächlich realisiere. Die von Sanders und Wurm vorgetragene Kritik hatte die öffentliche Meinung in einem Maße beeinflußt, das für Grimm bedrohlich zu werden schien. An den Tagebüchern des Zeitgeist-Chronisten Varnhagen von Ense ist abzulesen, wie seine anfängliche national motivierte Grimm-Begeisterung in kritische Reserviertheit umschlug, nachdem er von Sanders' Kritik Kenntnis erhalten hatte. Auch von Seiten des Verlegers waren immer wieder einmal kritische Bedenken an Grimm herangetragen worden, etwa bezüglich der zu zahlreichen Bibel-Belege. 23

Die Absicht, die Grimm mit seiner Vorrede verfolgte, mußte auf die Entkräftung der Kritik und die Verteidigung seiner Auffassung vom Nationalwörterbuch gerichtet sein. ${ }^{24}$ Die Thematik der sprachgeschichtlichen und lexikographischen Theoreme, auf die der Text in wissenschaftsgeschichtlichen Darstellungen oft genug reduziert worden ist, folgt nachweisbar den Vorgaben der Kritik.

23 Seit Kirkness (1980) Dokumentation liegt die Korrespondenz zwischen Grimm und Verleger vor.

24 Eine ausführliche Interpretation der Vorrede ist enthalten in meiner Habilitationsschrift über Daniel Sanders (Berlin 1995). 
Bezieht man die kommunikative Funktion wissenschaftsgeschichtlicher Quellentexte konsequent mit ein, relativiert sich auch ein bisher konstatierter qualitativer Unterschied zwischen wissenschaftlicher und nichtwissenschaftlicher Sprachreflexion. Der explizite theoretische Gehalt eines Textes ist nicht länger das alleinige Kriterium, das ihn zur historiographisch relevanten Quelle macht. Sprachwissenschaftsgeschichte besteht wesentlich darin, Sprachauffassungen auf interpretativem Weg aus den verschiedensten Quellentexten zu (re)konstruieren und dabei zwangsläufig auf ein höheres Abstraktionsniveau zu heben. Das Ergebnis von Interpretation und Abstraktion wird dann häufig ebenso als Theorie eingestuft wie heutige explizit ausgearbeitete theoretische Systeme. Die unterschiedliche Explizitheit theoretischer Systeme verdient gesondertes historiographisches Interesse, damit Theoriengeschichte zu den übrigen Formen von Sprachreflexion einer Sprachgemeinschaft hin offen bleiben und nicht von vornherein die Wechselwirkungen zwischen akademischer und allgemeiner Sprachreflexion ausgeblendet werden.

\section{Alternative Überlegungen}

Nachdem ich herausgearbeitet habe, aufgrund welcher prinzipiellen Entscheidungen über den Gegenstandsbereich und das vorausgesetzte Geschichtsbild der Fachgeschichtsschreibung der Germanistik ein Forscher wie Daniel Sanders interessant wird (oder nicht), und nachdem deutlich geworden ist, daß nur eine konsequent sozialhistorische Fundierung der Wissenschaftsgeschichte zu angemessenen Erklärungen führt, möchte ich einen noch weitergehenden Vorschlag zur fachgeschichtlichen Methode machen, der aus dem Versuch entstanden ist, die Hintergründe von Sanders' Werk und Sprachauffassung zu erklären.

Ich gehe zunächst davon aus, daß zwischen der Geschichte der Sprachwissenschaft und der Sprachgeschichte ein Zusammenhang besteht, insofern Sprachteilhaber über ihre Sprache reflektieren und kommunizieren können und dazu in jeder Sprache systematische, d. h. lexikalische, syntaktische und textuelle Ausdrucksmittel vorhanden bzw. ausbaubar sind. Eine bestimmte Gruppe der Sprachteilhaber kennzeichnet ihre Art von Sprachreflexion als wissenschaftlich, d. h. als eine eigens elaborierte Form. Wenn man den Ausdruck Sprachreflexion auf „die expliziten, den metasprachlichen Akt mit sprachlichen Mitteln andeutenden oder ausformulierenden Formen sprachlicher Reflexion“ (H. Schmidt 1988, 344) beschränkt und gewohnheitsmäßige reflektorische Rückkopplungsvorgänge während des Sprech- oder Schreibvorgangs (ebenda 345) sowie den unkommentierten Gebrauch performativer Ausdrücke, z. B. bestimmter Verben und Textsortenbezeichnungen, ausschließt, dann deckt Sprachreflexion einen Bereich von bloßer Thematisierung eines Teilaspekts von Sprache bis hin zu ihrer wissenschaftlich-systematischen Behandlung ab, ohne daß mit dem Basislexem Reflexion das Erfülltsein eines bestimmten Anspruchs an gedanklicher Durchdringung gekennzeichnet werden soll. Gardt et 
al. (1991) verwenden für die so eingegrenzte Bedeutung von Sprachreflexion den Terminus Meta-Reflexion. Sie gehen vom wechselseitigen Einfluß von Kommunikations- und Sprachgeschichte einerseits und Geschichte ihrer Reflexion andererseits aus und leiten daraus die Aufgabe der Sprachgeschichtsforschung ab, „die jeweils epochenüblichen Ausprägungen der Meta-Reflexion zu untersuchen" (ebenda 17).

Sprachreflexion in diesem Sinne schließt die Thematisierung des Verhältnisses ein, das eine historisch konkrete Sprachgemeinschaft zu ihrer Sprache bzw. zu ihren Sprachen hat. ${ }^{25}$ Thematisierung und z. T. kontroverse Diskussion dieses Verhältnisses in einer Sprachgemeinschaft, wie sie sich im 19. Jahrhundert beobachten läßt, ist zwangsläufig mit den herrschenden Vorstellungen von Kultur und Bildung verknüpft, die von Sprach- als auch von Wissenschaftshistorikern berücksichtigt werden müssen, insofern sich die Wissenschaft in irgendeiner Form beteiligt hat.

Im einzelnen handelt es sich um drei Faktorenkomplexe, die den Zusammenhang von Sprachgeschichte und Sprachwissenschaftsgeschichte konstituieren. Zum ersten Komplex gehören Sprachgemeinschaft, Sprachbewußtsein, unterschiedliche Formen der Sprachreflexion sowie Erarbeitung und Vermittlung von Sprachwissen. Der zweite Komplex wird gebildet vom Sprachsystem, seinem Entwicklungsstand und Potential in Beziehung zu den Problemen und Erfordernissen der gesellschaftlichen Kommunikation, wie sie aus politik-, sozial- und wirtschaftsgeschichtlichen Erkenntnissen abgeleitet werden können. Zum dritten Komplex gehören die gesellschaftlich verbreiteten Konzeptionen von Kultur und Bildung, die als „selbstgesponnene Bedeutungsgewebe“ verstanden werden können, mit dessen Hilfe soziale Gruppen sich ihre eigene Situation erklären (Geertz 1987, 22). Vor dem Hintergrund der kulturellen Konzepte erhalten sprachreflexive Handlungen erst eine bestimmte Funktion, und ihre Produkte werden zu Elementen des übergeordneten kulturellen Zeichensystems.

Ich möchte jetzt an diesen dritten Komplex anknüpfen und für die Hereinnahme eines kultursemiotischen Ansatzes in die Historiographie der Sprachreflexion plädieren.

\subsection{Kultur und Kulturkonzept}

Angesichts der Mannigfaltigkeit von Kulturbegriffen und ihrer Unschärfe soll hier zunächst der Gebrauch der Ausdrücke Kultur und Kulturkonzept geklärt werden.

Geertz $(1987,9)$ bestimmt Kultur als ein „,selbstgesponnenes Bedeutungsgewebe“ des sozial verfaßten Menschen, das den Interpretationsrahmen darstellt,

25 Hier ist vor allem an das Bewußtsein von der Existenz dialektaler oder funktionaler Varietäten neben der Standardvarietät gedacht. 
in dem sich eine soziale Gruppe die eigene Situation und die Umwelt deutet. Dieses Bedeutungsgewebe ist selbst nur auf interpretativem Weg erkennbar und beschreibbar. In der empirisch arbeitenden Geschichtswissenschaft wird Kultur mittlerweile ebenfalls

auf mental verankerte Orientierungsmuster und Verhaltensmaximen bezogen [...], die hinter authentischen kulturellen Objektivationen stehen. Kultur läßt sich begreifen als ein Rahmen, innerhalb dessen sich die Lebenspraxis handelnder, fühlender, denkender Menschen bewegt (K. Rohe zit. G. Schmidt 1986, 21).

Theoretisch exakter, aber weniger anschaulich formuliert besteht das selbstgesponnene Bedeutungsgewebe aus systematisch miteinander verbundenen zeichenhaften Elementen mit der geforderten Orientierungsfunktion. Die außerordentliche Komplexität dieses Zeichensystems wird von Geertz immer wieder mit Formulierungen wie „geschichtete Hierarchie bedeutungsvoller Strukturen“ (Geertz 1987, 12) betont. Drei Dimensionen sind für diese Komplexität verantwortlich:

(1.) Kulturelle Zeichen sind sozial festgelegt und nur innerhalb einer sozialen Einheit bedeutungsvoll. Die Sozialität haben kulturelle mit sprachlichen Systemen gemeinsam (vgl. G. Schmidt 1986, 21). Im Hinblick auf die konkrete Interpretation der für Sanders verbindlichen Kultur sei festgehalten, $\mathrm{da}$ das orientierende Gewicht eines kulturellen Zeichens abhängig ist vom Grad der Öffentlichkeit seiner Kommunizierbarkeit. Die soziale Dimension verweist ferner auf die Grenzen der Gültigkeit und die potentielle Mehrdeutigkeit kultureller Zeichen. Kulturelle Systeme verhalten sich ähnlich wie die Schichten einer Gesellschaft zueinander: Wirklichkeitsdeutung und Orientierung sind verschieden, aber dennoch kommunizierbar.

(2.) Kulturelle Zeichensysteme besitzen eine historische Dimension. ${ }^{26} \mathrm{Halb}$ wachs $(1985)^{27}$ folgend kann man von Kultur als ,kollektivem Gedächtnis“ sprechen (vgl. Assmann 1988). Diese Bestimmung ist insofern wichtig, als damit allen biologistischen Definitionsansätzen, die man bei der Redeweise von „mentaler“ Verankerung der Kultur assoziieren könnte, der Zugang verwehrt ist und werden soll. Um die Unterschiede und Gemeinsamkeiten in Weltdeutung von Deutschen und Juden, von christlichen Deutschen und jüdischen Deutschen im 19. Jahrhundert zu erkennen, ist ein Kulturmodell nötig, das die zeitgenössischen Modelle von Volk, Kon-

26 Der klassische Kulturbegriff war erstens ergologisch und zweitens soziativ bestimmt; seit Herder kam das historische Moment als drittes hinzu. Vgl. W. Perpeet, Kultur, Kulturphilosophie, in: Historisches Wörterbuch der Philosophie, Bd.4, Basel 1976: 1309-1323.

27 Das Buch des französischen Sozialwissenschaftlers jüdischer Herkunft, der 1945 im KZ Buchenwald ermordet wurde, entstand 1929 und erschien 1967 erstmals auf deutsch. 
fession oder Rasse überflüssig macht. Kulturelle Systeme lassen sich aber auch nicht auf traditionale Elemente reduzieren. Die Erfahrungen der jeweiligen Gegenwart formen und verändern die tradierten Deutungsangebote (vgl. G. Schmidt 1986, 22).

(3.) Die ausschließlich interpretative Zugänglichkeit von Kultur macht methodisch bewußtes Vorgehen notwendig. Durch sie kann eine Methode legitimiert werden, mit der repräsentative kulturelle Zeichen in den zugänglichen Quellen und in Form sprachlicher Ausdrücke aufgesucht und ihre semantischen Relationen untereinander als anschaulicher Teil des kulturellen Gesamtsystems dargestellt werden. Der semiotische Kulturbegriff erlaubt es z. B., Wörterbücher, Formulierungskonstanten in ihren Bedeutungserläuterungen, Wörterbuch-Vorreden und alle möglichen lexikographischen Entscheidungen ebenso als zeichenhaft zu behandeln, wie die aus Briefen, Zeitschriftenartikeln und sonstigen sprachdidaktischen Texten rekonstruierbaren Leitideen. Leitideen nenne ich die interpretativ gewonnenen Elemente des kulturellen Zeichensystems, dessen sich historisch interessierende Individuen in Übereinstimmung mit einer bestimmten Gruppe der bürgerlichen Gesellschaft bedienten. Das Ensemble der Leitideen bildet das Kulturkonzept. Mit Kulturkonzept beziehe ich mich auf das interpretativ gewonnenen Konstrukt des $z$. T. selbstgesponnenen, $z$. T. tradierten Bedeutungsgewebes, in dem Gruppen und Individuen leben. Der Ausdruck Kulturkonzept und nicht etwa -theorie, -begriff, -verständnis u.ä. wird hier verwendet, weil er die größtmögliche Distanz zu den Ausdrucksformen des (zeitgenössischen) Selbstverständnisses zu garantieren scheint. Es geht bei diesem Ausdruck nicht um das, was ein Sprachforscher des 19. Jahrhunderts selbst unter Kultur verstand. Von Weltbild wird Kulturkonzept unterschieden durch seine bewußte, einschränkende Ausrichtung auf diejenigen semiotischen Elemente, die den Rahmen für die Auffassung von Sprache, Sprachbildung und Lexikographie bilden.

Wie verhält sich der kultursemiotische Ansatz zur sogenannten MentalitätenGeschichte ${ }^{28}$, die inzwischen auch in literatur- und sprachhistorischen Arbeiten immer öfter, wenn z. T. auch nur als wissenschaftliches Schlagwort zitiert wird? In der Mentalitäten-Geschichte wird der Bereich des Mentalen in Abgrenzung zum Bereich der Ideen und des Ideologischen bestimmt. Ihr Gegenstand ist kulturpsychologischer, nicht ideengeschichtlicher oder gar philosophischer Art; ihr geht es um die präreflexiv-affektiven Orientierungen, um die Schicht der tendenziell eher unbewußten Glaubensgewißheiten (vgl. Raulff 1987, 7-15). Zwar ist auch der kultursemiotische Ansatz kein rein ideengeschichtlicher, denn es werden nicht nur explizit geäußerte Gedankeninhalte und -systeme berücksichtigt, sondern auch die Glaubensgrundsätze, die beiläufig, aber wiederkehrend angeführten Wertkategorien, Lebensziele, Autoritäten, wie 
sie in Metaphern und Symbolen erfaßbar sind. Dennoch tendiert der kultursemiotische Ansatz mehr zu den bewußten als zu den unbewußten Orientierungen. Burke (in Raulff $1987,137 \mathrm{ff}$ ) hat für die Öffnung der Mentalitäten-Geschichte gegenüber sozialwissenschaftlichen Fragen und für begrifflich erfaßbare Kategorien plädiert, weil „erst die Interessenkonflikte das Unbewußte bewußt und das Implizite explizit [machen] und auf diese Weise zu Veränderungen [führen]“ (Burke in Raulff 1987, 137). Die Mentalitäten-Geschichte nähert sich damit an den linguistischen Gegenstandsbereich an, während das kultursemiotische Interesse sich von vornherein entschieden dem sprachlich realisierten Bereich zuwendet, und zwar nicht nur aus Gründen der Quellenverfügbarkeit, sondern aus der grundsätzlichen Überzeugung, daß Orientierungen (,Bedeutungsgewebe“) von Menschen nur als Interpretationsergebnisse von irgendwelchen realisierten Kommunikations- und Ausdrucksformen erkannt werden können.

\subsection{Das Kulturkonzept bei Daniel Sanders}

Ich habe nun nach der Sprachauffassung von Daniel Sanders und nach den Motiven und Zielen seines sprachreflexiven Handelns gefragt, indem ich in seinen diversen Texten, d. s. Wörterbuchartikel ebenso wie Zeitschriftenartikel und Briefe, nach Leitideen gesucht habe: nach den Letztbegründungen in Argumentationsketten, nach den Inhalten vor allem der denn- und weil-Sätze, nach metaphorischen Konzepten sowie nach Wertbegriffen. Das kulturelle System oder Kulturkonzept, in dem Sanders sich bewegte, war einmal das des aufgeklärten liberalen deutschen Judentums, zum zweiten das des gebildeten Bürgertums. Zentrale Leitideen seiner sprachreflexiven und politischen Arbeit waren: Bildung, besonders in der Form der Selbstbildung, und Aufklärung. Diesen beiden Säulen lassen sich weitere Leitideen zuordnen: Das Individuum und seine Entwicklungsfähigkeit durch Bildung; eine von gesellschaftlicher Nützlichkeit her gedachte Bildung; die Polarisierung von Herzensbildung gegenüber falscher Gelehrsamkeit (Gelahrtheit), mit der Sanders ein undemokratisches Bildungskonzept wie z. B. das von Jacob und Wilhelm Grimm kennzeichnete. Aus dem politischen Prinzip der Volkssouveränität leitete Sanders die Auffassung ab, der tatsächliche Sprachgebrauch sei die oberste Instanz in allen Fragen der sprachlichen Norm. Die Aufklärung lieferte ihm das bei weitem beliebteste Metaphernfeld. Die historische Aufklärungsbewegung bedeutete ihm auch den Hauptidentifikationsansatz mit der deutschen Kultur. Goethe, Lessing, Schiller, in dieser Reihenfolge, waren ihm Gewährsmänner und Sprachrohre zugleich. Gemäß seiner jüdischen Geschichtserfahrung orientierte Sanders sich an Kulturkontakt im europäischen Rahmen als einem Moment der gegenseitigen Befruchtung, das die antike Konstellation von Hellenismus, Judentum und Römerreich zum Vorbild hat. Von der Idee der Aufklärung her erwuchs auch der hohe Stellenwert der Zeitgemäßheit und des Fortschritts der Geschichte als beständigem Zuwachs an Zivilisation und Humanität. 


\subsection{Eine Lexikographie des „Fortschritts“}

Abschließend möchte ich an einer der genannten Leitideen, der der Zeitgemäßheit und des Fortschritts, exemplarisch zeigen, wie sich das Kulturkonzept in einem Teilbereich sprachreflexiver Tätigkeit, nämlich der Lexikographie widerspiegelt.

Sanders' Zeitbegriff beeinhaltete das Bewußtsein von der Aufwärtsentwicklung aller zivilisatorischen Verhältnisse: Die Bewegungsrichtung der Zeit, der Politik, der Gesellschaft und auch der Sprache ist Fortschritt, nicht Rückschritt. In seinem 1899 postum erschienenen Citatenlexikon läßt er Börne Zeit definieren als „nämlich die Mehrzahl der gleichzeitig lebenden Menschen in einem Staate“ (S.702). Sanders' Bewußtsein, von den Bedingungen seiner Zeit abzuhängen, war sehr ausgeprägt, denn als Jude erlebte er die Spannung des Übergangs von der ständischen zur bürgerlichen Gesellschaft unmittelbarer als andere. Mehrere lexikographische Entscheidungen sind nachweislich an der Leitidee des Fortschritts ausgerichtet:

Zunächst die Wahl der Wörterbuchquellen. Daß Sanders die Autoren des Jungen Deutschland und Erfolgsschriftsteller der Jahrhundertmitte ebenso ausführlich berücksichtigt und gleiches auch von der Grimmschen Lexikographie gefordert hatte, war ihm von der historischen Richtung übel angekreidet worden. Für Sanders war jedoch nicht der Maßstab literarischer Qualität ausschlaggebend, sondern die Nähe dieser Literatur zu derjenigen Varietät, die seiner Vorstellung gemäß lexikographisch zu bearbeiten war: der Sprachgebrauch seiner Gegenwart. Unter dem Stichwort neu (1.d) seines Wörterbuchs der deutschen Sprache nimmt er auf dieses Spezifikum seiner Wörterbuchbasis Bezug:

„Die neue Zeit, im Ggstz. zur alten die Gegenwart und die ihr nächste Vergangenheit

[...] Die neuern [der neuern Zeit angehörigen] Schriftsteller“" (WDS II.1, 431).

Zweitens zeigt sich die Leitidee des Fortschritts in der Auswahl der Stichwörter. Zum Sprachgebrauch der Gegenwart zählt er erstens selbstverständlich die gemeinsprachnahe Terminologie der Wissenschaften und der Technik, insbesondere der Wirtschaft und der Verwaltung. Zeitgemäß und entsprechende Paraphrasen sind auch in Sanders' nicht-lexikographischen Schriften ausgesprochen häufig. Etwa bewertet er einem Briefpartner gegenüber die Aufnahme technischer und gewerblicher Ausdrücke ins Wörterbuch als ,durchaus zeitgemäß“" ${ }^{29}$ Die Begründung für das 1885 erschienene Ergänzungswörterbuch der deutschen Sprache greift auf dieselbe Idee zurück:

Im Jahre 1860 habe ich mein großes dreibändiges ,Wörterbuch der deutschen Sprache" abgeschlossen. Wie viele neuen Wörter und Ausdrücke sind seit jener Zeit mit dem Aufschwunge und den Fortschritten unseres Staats-, Verfassungs- und Rechtslebens, unseres Heer- und unseres Verkehrswesens, und auf den Gebieten der Erfin-

29 Brief an Joachim Meyer v. 4.6.1859. Deutsches Literaturarchiv Marbach/Cotta-Archiv. 
dungen und Entdeckungen entstanden! (Vorwort Ergänzungswörterbuch datiert 3.10.1884, S.I; Hervorhebung U. H.-Z.)

Ferner versuchte Sanders, auch den auffälligen systematischen Veränderungen des Wortschatzes im 19. Jahrhundert Rechnung zu tragen. Um der steigenden Produktivität und Flexibilität in der Wortbildung lexikographisch gerecht zu werden, wählte er die für den Benutzer nicht unproblematische Lemmatisierung nach dem Grundwort. Danach erscheinen Fortschritt und Rückschritt nebeneinander als Sublemmata unter Schritt, ferner die Sublemmata nächster Ordnung Geistesfortschritt, Kulturfortschritt und Weltfortschritt neben dem Kollokationsbeispiel „Fortschritte in den Wissenschaften“ (WDS II.2, 1015). Nur diese lexikographische Methode scheint ihm geeignet, die Fülle mehr oder weniger lexikalisierter und durchsichtiger Zusammensetzungen $\mathrm{zu}$ bewältigen, die durch syntaktische Komprimierung vor allem in Zeitungen und Zeitschriften gebildet werden. Z. B.:

Entwicklung [...] Alle Fortentwicklung der Menschheit. Fichte [...]; Noch entsprach das Ständewesen der damaligen Gesammtentwickelung. Gartenl. [...]; Die ersten Stufen der modernen Gesellschaftsentwicklung. Gneist [...]; Geistesentwicklung [...], Ideenentwicklung [...], Kulturentwicklung. Natur Beil. (WDS II.2, 1596f)

Drittens spricht Sanders' eigener Glaube an eine positive Weiterentwicklung der Menschheitsgeschichte aus vielen seiner Bedeutungsangaben, seinen selbstgebildeten (Kompetenz-)Beispielen und aus der Wahl der zitierten Beispielbelege. Geschichte paraphrasiert Sanders als

die zusammenhängende Darstellung Dessen, worin sich die fortschreitende Entwicklung eines großen Ganzen bekundet, [...] bes. von der Entwicklung der Menschen, im engsten Sinn gew. vom polit. Standpunkt aus [...] Die Geschichte der Menscheit, eines Volks, Staats, der Juden, Griechen, Deutschen [...] (WDS II.2, 913 unter 4.)

Deutlicher noch wird seine Auffassung in den Charakterisierungen der Gegenwart:

Zeit [...] 30.a) in Bezug auf die Zeit-Verhältnisse, für die in einer gewissen Zeit Lebenden [...]; Die finstern Zeiten des Mittelalters; Die aufgeklärte Zeit unsres Jahrhunderts; [... 31.c) = Zeitalter; das darinlebende Geschlecht, die Generation; der Geist, der in dem Geschehenden sich als das die Generation Bewegende kundgiebt [...]; Dass nicht ihr stets Altvordere rühmend erhöhn mögt/.. und die jüngere Zeit darstellt als weltlich und gottlos [...] Platen [...]; Die Gesetze müssen sich den Sitten und die Maximen den Zeiten anschmiegen. Schiller [...] Überbleibsel aus der Feudalzeit. Gartenlaube [...] Zeit des Feudalwesens [...] Wir Leute der Jetztzeit. Magazin der Lit. d. Auslands. (WDS II.2, 1723f)

„Zeit“ war auch ein wesentlicher Bestandteil des politischen Vokalbulars der Linken in der Frankfurter Nationalversammlung. Als semantische Äquivalente wurden Gegenwart, Neuzeit, Jetztzeit verwendet, die zusammen mit weiteren Ausdrücken (demokratisch, zeitgemäß, Geist der Zeit, Zeitgeist, öffentliche Meinung) ein zentrales Begriffsfeld dieser politischen Gruppe bildeten (Grünert 1974, 230ff, 244f). Stigmatisierende Antonyme waren, ähnlich wie in Sanders' Wortartikel, Mittelalter sowie Reaktion und Romantik (Wülfing 1982). 
Das Aufspüren bestimmter Leitideen in solchen Kommentaren darf nicht als Enthüllung eines lexikographischen Subjektivismus mißverstanden werden. Stärker als die älteren Bearbeiter des Grimmschen Wörterbuchs bemüht sich Sanders um weltanschauliche Ausgewogenheit. Auch dies kann mit seiner Erfahrung, einer gesellschaftlichen Minderheit anzugehören, in Zusammenhang gebracht werden: Die Kenntnisse der Mehrheitskultur standen bei ihm neben denen seiner eigenen jüdischen Teilkultur. Sanders' Bedeutungsangaben sind durch ein Nebeneinander verschiedener Perspektiven gekennzeichnet:

Neuern: Etwas, weil es Einem veraltet erscheint, so umgestalten, wie man es der Neu-, der Jetztzeit gemäß erachtet, oft mit tadelndem Nebenbegriff ungerechtfertigter Missachtung des Alten, Bestehenden (WDS II.1, 433)

Den deutschen Bildungsbürgern des späten 19. Jahrhunderts traten manche ihrer ideologischen Fahnenwörter - etwa Deutsch, Deutschthum, Volk und Staat - aus Sanders' Wörterbuch perspektivisch relativiert und distanziert entgegen. Wie kein anderer Lexikograph arbeitete er subtile Wertungen und verdeckte Maßstäbe des Wortgebrauchs heraus und verbalisierte sie. Vielleicht ist die heutige Zeit, anders als die seine, in der Lage, diesen Zugewinn an Sprachreflexion zu verstehen und auch zu würdigen, obwohl die Geschichte entgegen der Überzeugung des deutschen Juden Daniel Sanders im 20. Jahrhundert keinen Zuwachs an Humanität aufzuweisen hat.

\section{Literatur}

Arens, Hans: Sprachwissenschaft. Der Gang ihrer Entwicklung von der Antike bis zur Gegenwart, 2 Bde. Frankfurt 1974 (= Taschenbuchausgabe, identisch mit der 2. Aufl. 1969).

Assmann, Jan: Kollektives Gedächtnis und kulturelle Identität. In: Kultur und Gedächtnis, hrsg. von Jan Assmann und Tonio Hölscher, Frankfurt/M. 1988, 9-19.

Bahner, Werner (Hg.): Sprache und Kulturentwicklung im Blickfeld der deutschen Aufklärung. Der Beitrag Johann Christoph Adelungs. Berlin 1984. (Abhandlungen der Sächsischen Akademie der Wissenschaften Leipzig, Phil.-hist. Klasse Bd. 70, H. 4). (1984a)

Bahner, Werner: Johann Christoph Adelung (1732-1806). Zum historischen Stellenwert seines wissenschaftlichen und publizistischen Wirkens. In: Bahner 1984a, 7-24. (1984b)

Bahner, Werner / Neumann, Werner (Hgg.): Sprachwissenschaftliche Germanistik. Ihre Herausbildung und Begründung, Berlin (Akad. d. Wiss. der DDR/ ZISW) 1985.

Besch, Werner / Reichmann, Oskar / Sonderegger, Stefan (Hgg.): Sprachgeschichte. Ein Handbuch zur Geschichte der deutschen Sprache und ihrer Erforschung. 2 Halbbde., Berlin/New York 1984; 1985. (Handbücher zur Sprach- und Kommunikationswissenschaft 2,1; 2,2). 
Dutz, Klaus D. / Schmitter, Peter (Hgg.): Geschichte und Geschichtsschreibung der Semiotik. Fallstudien, Münster 1986.

Ebel, Else: Jacob und Wilhelm Grimm und ihre Vorlesungstätigkeit in Göttingen 1830-1837. In: Brüder Grimm Gedenken Bd. 4, hrsg. von Ludwig Denecke, Marburg 1984, 56-98.

Eggers, Hans: Deutsche Sprachgeschichte Bd. IV (Das Neuhochdeutsche), Reinbek 1977.

Emmerich, Wolfgang: Zur Kritik der Volkstumsideologie, Frankfurt 1971.

Engster, Herrmann: Germanisten und Germanen. Germanenideologie und Theoriebildung in der deutschen Germanistik und Nordistik von den Anfängen bis 1945, Frankfurt/M. 1986.

Gardt, Andreas / Lemberg, Ingrid / Reichmann, Oskar / Roelcke, Thorsten: Sprachkonzeptionen in Barock und Aufklärung. Ein Vorschlag für ihre Beschreibung, In: ZPSK 44/1991, 17-33.

Geertz, Clifford: Dichte Beschreibung. Beiträge zum Verstehen kultureller Systeme, Frankfurt/M. 1987.

Gipper, Helmut / Peter Schmitter: Sprachwissenschaft im Zeitalter der Romantik, Tübingen 1985.

GreB, Franz: Germanistik und Politik. Kritische Beiträge zur Geschichte einer nationalen Wissenschaft. Stuttgart-Bad Cannstatt 1971.

Grimm, Jacob: Kleinere Schriften, Bd. VIII, Gütersloh 1890.

Grünert, Horst: Sprache und Politik. Untersuchungen zum Sprachgebrauch der Paulskirche, Berlin/New York 1974.

Halbwachs, Maurice: Das kollektive Gedächtnis, Frankfurt/M. 1985.

Haß-Zumkehr, Ulrike: Daniel Sanders. Aufgeklärte Germanistik im 19. Jahrhundert. Berlin/New York 1995. (Studia Linguistica Germanica 35).

Helbig, Gerhard: Geschichte der neueren Sprachwissenschaft. München 2. Aufl. 1973.

Ivo, Hubert: Jacob Grimm und die sprachdidaktischen Häresien, In: DD 20/ 1989-90:586-593.

Jäger, Ludwig: Philologie und Linguistik. In: Schmitter, Peter (Hg.): Zur Theorie und Methode der Geschichtsschreibung der Linguistik, Tübingen 1987. (Geschichte der Sprachtheorie 1), 198-223.

Janota, Johannes (Hg.): Eine Wissenschaft etabliert sich. 1810-1870, Tübingen 1980.

Katznelson, Siegmund: Juden im deutschen Kulturbereich, 2. stark erweit. Ausgabe Berlin 1959.

Kirkness, Alan: Geschichte des Deutschen Wörterbuchs 1838-1863, Stuttgart 1980.

Kirkness, Alan: Die nationalpolitische Bedeutung der Germanistik im 19. Jahrhundert, Ersetzt statt erforscht - Thesen zu Lehndeutsch, Purismus und Sprachgermanistik. In: Das 19. Jahrhundert. Jahrbuch 1990 des Instituts für deutsche Sprache, hrsg. v. R. Wimmer, Berlin 1991, 294-306.

Knobloch, Clemens: Zeichen und Bild bei Gustav Gerber und Ludwig Noiré. Ein Beitrag zur Geschichte der Semantiktheorie im 19. Jahrhundert. In: 
Dutz/Schmitter 1986, 163-180.

Monreal-Wickert, Irene: Die Sprachforschung der Aufklärung im Spiegel der großen französischen Enzyklopädie, Tübingen 1977.

Müller, Jörg Jochen (Hg.): Germanistik und deutsche Nation 1806-1848. Zur Konstitution bürgerlichen Bewußtseins. Stuttgart 1974.

Neumann, Werner: Über das Verhältnis von Sprachtheorie und Sprachsituation in Deutschland gegen Ende des 19. Jahrhunderts. In: Fleischer, Wolfgang / Rudolf Große / Gotthard Lerchner (Hgg.): Beiträge zur Erforschung der deutschen Sprache, Bd.8, Leipzig 1988, 5-33.

Neumann, Werner / Schmidt, Hartmut (Hgg.): Jacob Grimm. Reden in der Akademie, Berlin 1984.

Polenz, Peter v.: Das 19. Jahrhundert als sprachgeschichtliches Periodisierungsproblem. In: Cherubim, Dieter / Mattheier, Klaus (Hgg.): Voraussetzungen und Grundlagen der Gegenwartssprache. Sprach- und sozialgeschichtliche Untersuchungen zum 19. Jahrhundert, Berlin/New York 1989, 11-30.

Putschke, Wolfgang: Die Arbeiten der Junggrammatiker und ihr Beitrag zur Sprachgeschichtsforschung, In: Besch/Reichmann/Sonderegger 1984, 331-347.

Raulff, Ulrich (Hg.): Mentalitäten-Geschichte. Zur historischen Rekonstruktion geistiger Prozesse, Berlin 1987.

Reichmann, Oskar: Erbwortbezogene Wörterbücher im Deutschen. In: Wörterbücher. Ein internationales Handbuch zur Lexikographie, hrsg. von Franz Josef Hausmann, Oskar Reichmann, Herbert Ernst Wiegand, Ladislav Zgusta, 2. Teilbd., Berlin/New York 1990, 1231-1241.

Rüsen, Jörn: Historische Vernunft. Göttingen 1983.

Sanders, Daniel: Wörterbuch der deutschen Sprache. Mit Belegen von Luther bis auf die Gegenwart, 2 in 3 Bde, 2. unveränd. Aufl. Leipzig 1876 (nachgedruckt Hildesheim 1969).

Schmidt, Gustav: Gelehrtenpolitik und politische Kultur in Deutschland - Zur Einführung. In: ders. und Jörn Rüsen (Hgg.): Gelehrtenpolitik und politische Kultur in Deutschland 1830-1930, Bochum 1986, 5-37.

Schmidt, Hartmut: Sprachkompetenz und Sprachreflexion als Probleme der historischen Semantik, in: Sprachwissenschaft 13/1988, 334-355.

Schmitter, Peter: Untersuchungen zur Historiographie der Linguistik, Tübingen 1982.

Schmitter, Peter: Das sprachliche Zeichen. Studien zur Zeichen- und Bedeutungstheorie in der griechischen Antike sowie im 19. und 20. Jahrhundert, Münster 1987.

Varnhagen von Ense, K. A.: Tagebücher aus dem Nachlaß, 1.-11. Bd., Leipzig, 1861-1869.

Vesper, Wilhelm: Deutsche Schulgrammatik im 19. Jahrhundert. Zur Begründung einer historisch-kritischen Sprachdidaktik, Tübingen 1980.

Wolff, Gerhart: Deutsche Sprachgeschichte, Frankfurt/M. 1986.

Wülfing, Wulf: Schlagworte des Jungen Deutschland, Berlin 1982.

Wyss, Ulrich: Die wilde Philologie, München 1979. 